\title{
Factors associated with mothers' hesitancy to receive a COVID-19 vaccine
}

\author{
Molly E. Waring ${ }^{1} \odot$ - Sherry L. Pagoto ${ }^{1}$ Lauren R. Rudin ${ }^{1} \cdot$ Chloe Ho $^{1} \cdot$ \\ Alexa Horkachuck ${ }^{1}$ Indra A. Kapoor ${ }^{1} \cdot$ Quamyia Foye $^{1}$
}

Received: 1 July 2021 / Accepted: 21 November 2021 / Published online: 4 January 2022

(C) The Author(s), under exclusive licence to Springer Science+Business Media, LLC, part of Springer Nature 2021

\begin{abstract}
Vaccine hesitancy can impact maternal and child vaccination rates. We examined factors associated with mothers' hesitancy to receive a COVID-19 vaccine using data from an online survey conducted from midFebruary to mid-March 2021. Among unvaccinated participants $(\mathrm{N}=203), 28 \%$ reported that they would probably not or definitely not get a COVID-19 vaccine. Mothers with high school/GED/trade/technical education (38\% hesitant, $\mathrm{aOR}=4.0,95 \%$ CI: $1.2-13.2)$, Associate's degree (43\%, $\mathrm{aOR}=6.8,95 \% \mathrm{CI}: 2.4-19.5)$, and Bachelor's degree (30\%, $\mathrm{aOR}=3.1,95 \% \mathrm{CI}: 1.1-8.4)$ were more likely to report vaccine hesitancy compared to mothers with a graduate degree (19\%). Non-Hispanic Black mothers (40\% hesitant, $\mathrm{aOR}=2.8,95 \% \mathrm{CI}: 1.0-7.6)$ were more likely to be vaccine hesitant compared to non-Hispanic white mothers (19\%). Mothers with low pandemic-related anxiety were more likely to report vaccine hesitancy than mothers with high pandemic-related anxiety (56\% vs $23 \%$ hesitant; aOR $=4.8$, 95\% CI: 1.7-14.1). Research is needed to understand informational, emotional, and attitudinal factors contributing to COVID-19 vaccine hesitancy among mothers to develop and test effective public health messaging to increase vaccination rates.
\end{abstract}

Keywords COVID-19 - Vaccine hesitancy · Mothers . Education

Molly E. Waring

molly.waring@uconn.edu

1 Department of Allied Health Sciences and UConn Center for mHealth and Social Media, University of Connecticut, 358 Mansfield Rd, Unit 1101, Storrs, CT 06269, USA

\section{Introduction}

Vaccines are a critical tool to curb the COVID-19 pandemic. However, by mid-April 2021, only $45 \%$ of US adults had received at least one dose of a COVID-19 vaccine (Murthy et al., 2021). Over the previous year, studies have documented COVID-19 vaccine hesitancy rates upwards of a quarter to half of US adults [e.g., 49\% in September 2020 (Funk \& Tyson, 2021), 32\% in December 2020 (Nguyen et al., 2021), and 30\% in mid-February 2021 (Funk \& Tyson, 2021)]. A survey of US parents in spring 2021 found that those who had received or intended to receive a COVID-19 vaccine were more than three times as likely to intend to vaccinate their children (Szilagyi et al., 2021). As nearly $80 \%$ of mothers report being the parent primarily responsible for making decisions about their children's health (Gomez, et al., 2018), understanding factors associated with COVID-19 vaccine hesitancy among mothers could inform public health messaging to increase COVID-19 vaccination rates not only among US mothers, but also among their children. In this study, informed by previous research examining COVID-19 vaccination receipt and intentions, (Head, et al., 2020; Khubchandani et al., 2021; Nguyen et al., 2021; Skjefte et al., 2021) we examined demographic (e.g., age, education), clinical (e.g., pregnancy), and COVID-19-specific (e.g., infection history, pandemic-related anxiety) factors associated with mothers' hesitancy to receive a COVID19 vaccine. 


\section{Methods}

\section{Participants and procedures}

This study is a secondary analysis of data from an online cross-sectional survey of mothers conducted from midFebruary to mid-March 2021. Eligible participants were 18 years or older, identified as a mother of child(ren) aged 0-12 years, posted or replied on Facebook, Instagram, and/ or Twitter in the last 7 days (the parent study aimed to examine how mothers respond to child nutrition-related misinformation on social media), live in the US, able to complete the survey in English or Spanish, and willing to provide informed consent. We recruited participants on ResearchMatch (Harris et al., 2012). To increase the diversity of our sample, we searched for potential participants stratified by race/ethnicity. Participants were sent a reminder one day after the initial survey invitation. The survey was administered through Qualtrics (Qualtrics LLC, Provo, UT). Participants who completed the survey received a \$20 Amazon gift card. The University of Connecticut Institutional Review Board approved this study. Participants provided informed consent.

\section{Measures}

We asked participants if they had received a COVID-19 vaccine. Participants who had not yet received a COVID-19 vaccine were asked once a vaccine to prevent COVID-19 is available to them, would they get the vaccine (US Census Bureau, 2021). We combined responses of "probably not get the vaccine", and "definitely not get the vaccine" to represent vaccine hesitancy (vs responses of "definitely get the vaccine" and "probably get the vaccine").

Participants self-reported their age, Hispanic ethnicity, race, and other demographic characteristics. We categorized race/ethnicity as non-Hispanic white, non-Hispanic Black, Hispanic/Latina (any race[s]), non-Hispanic Asian, and nonHispanic other race/multiracial. Mothers reported their education attainment; no participants reported less than a high school education, and we collapsed "high school graduate or GED" ( $\mathrm{n}=17)$ and "post high school trade or technical education" $(n=9)$. Mothers reported the number of children $0-5$ years and adults 65 years or older in their household (both dichotomized as $1+$ vs 0 ). We asked mothers, "How confident are you filling out medical forms by yourself?" and considered responses of not at all, a little bit, or somewhat confident to indicate impaired health literacy and quite a bit or extremely confident to indicate adequate health literacy (Powers, et al., 2010). Mothers reported whether they lived in a large city, a suburb near a large city, a small city or town, or a rural area; we dichotomized responses due to the small number living in rural areas $(\mathrm{n}=13)$.

Participants reported whether they had tested positive for COVID-19, whether any close family member tested positive for COVID-19, whether they personally know anyone who has died of COVID-19, whether they had any physical or mental health conditions that require regular medical visits, and whether they were currently pregnant. We combined women who selected "unsure" $(n=4)$ regarding pregnancy with those reporting that they were pregnant. We measured anxiety about the COVID-19 pandemic using the disease anxiety subscale of the pandemic anxiety scale (PAS) (McElroy et al., 2020) which includes Likert scale items that assess the degree of agreement with the following statements: "I'm worried that I will catch COVID-19," "I'm worried that my family and friends will catch COVID-19," "I' $m$ afraid to leave the house right now," and "I'm worried I might transmit COVID-19 to someone else." We summed responses to create a total score (possible range: $0-16$ ) and categorized pandemic-related anxiety as low ( $0-4$, equivalent to "strongly disagree" or "disagree" on all 4 items), lowto-medium (5-8), medium-to-high (9-11) or high (12-16, equivalent to "strongly agree" or "agree" on all 4 items).

\section{Statistical analysis}

We described characteristics of the sample overall and by maternal characteristics. We used logistic regression models to estimate odds of vaccine hesitancy by maternal characteristics. We included in a preliminary adjusted model characteristics for which at least one level of the variable was associated $(\mathrm{p}<0.05)$ with vaccine hesitancy in crude models, and retained characteristics associated $(\mathrm{p}<0.05)$ with vaccine hesitancy in the final adjusted model (bolded). Analyses were conducted in SAS 9.4 (SAS Institute, Inc., Cary, NC).

\section{Results}

When surveyed in mid-February to mid-March 2021, 31\% $(n=96)$ of mothers had already received at least one dose of a COVID-19 vaccine. Of unvaccinated participants, we excluded those missing vaccine intentions $(n=1)$ or any characteristics examined $(n=5)$, resulting in an analytic sample of 203 mothers. Participants were on average 35.9 (SD: 6.7) years old, $26 \%$ were non-Hispanic white, and $67 \%$ had at least a Bachelor's degree. Participants resided in 32 US states and Washington, DC. Additional participant characteristics are shown in Table 1.

Almost 3 in 10 unvaccinated mothers $(28 \%, \mathrm{n}=56)$ reported that they would probably not or definitely not get the COVID-19 vaccine $(10 \%, \mathrm{n}=21$ definitely not get, 
Table 1 Characteristics associated with COVID-19 vaccine hesitancy among mothers of children 0-12 years who have not yet received a COVID-19 vaccine

Overall sample, Probably or definitely not get the vaccine

$\mathrm{N}(\%) \quad$ Crude OR (95\% CI) $\quad$ Adjusted OR (95\% CI)

$$
\begin{aligned}
& \text { Age (years) } \\
& \text { 18-29 years } \\
& \text { 30-34 years } \\
& \text { 35-39 years } \\
& \text { 40-55 years }
\end{aligned}
$$

Race/ethnicity

Non-Hispanic White

Non-Hispanic Black

Hispanic/Latina (any race)

Non-Hispanic Asian

Non-Hispanic other race or multiracial

Education

HS, GED, trade or technical

Associates degree

Bachelor's, some graduate

Graduate degree

Health literacy

Adequate
Impaired

Place of residence

A large city or a suburb

A small city/town or rural

Currently pregnant

No

Yes/unsure

$0-5$ year olds in household

None
One or more children

$65+$ year olds in household

None

One or more

Tested positive for COVID-19

Yes

No

Close family member tested positive for COVID-19

Yes

$63(31)$

$140(69)$

$25(40)$

$31(22)$

Know someone who died of COVID-19

$\begin{array}{lrr}\text { Yes } & 75(37) & 18(24) \\ \text { No } & 128(63) & 38(30)\end{array}$

Has physical or mental health condition that requires regular medical visits

\begin{tabular}{lrcc} 
Yes & $63(31)$ & $20(32)$ & $1.3(0.7-2.6)$ \\
No & $140(69)$ & $36(26)$ & (Reference) \\
Pandemic anxiety scale & & & \\
Low (0-4) & $27(13)$ & $15(56)$ & $\mathbf{4 . 2}(\mathbf{1 . 6 - 1 1 . 3 )}$ \\
Low-to-medium (5-8) & $57(28)$ & $16(28)$ & $1.3(0.6-3.1)$ \\
Medium-to-high (9-11) & $62(31)$ & $12(19)$ & $0.8(0.3-2.0)$ \\
\hline
\end{tabular}

$1.5(0.6-3.4)$

(Reference)

(Reference)

$1.8(0.9-3.4)$

(Reference)

$1.3(0.4-4.1)$

(Reference)

$0.7(0.4-1.4)$

(Reference)

$0.3(0.1-1.3)$

$1.2(0.5-2.9)$

(Reference)

2.3 (1.2-4.4)

(Reference)

$0.7(0.4-1.4)$

(Reference)

$0.8(0.3-2.0)$
$1.0(0.4-2.5)$

$1.4(0.6-3.1)$

0.7 (0.3-1.7)

(Reference)

(Reference)

(Reference)

2.7 (1.1-6.9)

2.8 (1.0-7.6)

2.7 (1.1-6.7)

$2.3(0.8-6.4)$

$0.9(0.2-3.2)$

$1.4(0.4-5.7)$

$0.9(0.3-3.0)$

4.7 (1.6-13.8)

$4.0(1.2-13.2)$

$5.6(2.2-14.7)$

$6.8(2.4-19.5)$

3.2 (1.3-7.9)

3.1 (1.1-8.4)

(Reference)

(Reference)
4.8 (1.7-14.1)

$1.6(0.6-4.0)$

$0.9(0.4-2.4)$ 
Table 1 (continued)

\begin{tabular}{llllll}
\hline & Overall sample, & \multicolumn{3}{l}{ Probably or definitely not get the vaccine } \\
\cline { 3 - 6 } & $\mathrm{N}(\%)$ & $\mathrm{N}(\%)$ & Crude OR (95\% CI) & Adjusted OR (95\% CI) \\
\hline High (12-16) & $57(28)$ & $13(23)$ & (Reference) & (Reference) \\
\hline
\end{tabular}

$17 \%, \mathrm{n}=35$ probably not get, $32 \%, \mathrm{n}=65$ probably get, $40 \%, \mathrm{n}=82$ definitely get). Across maternal characteristics, vaccine hesitancy ranged from $11 \%$ among participants living with an adult aged $65+$ years to $56 \%$ among mothers with low pandemic-related anxiety (Table 1). Mothers with a high school education/GED or trade or technical education (38\% hesitant, aOR $=4.0,95 \% \mathrm{CI}: 1.2-13.2)$, Associate's degree $(43 \%, \mathrm{aOR}=6.8,95 \% \mathrm{CI}: 2.4-19.5)$, and Bachelor's degree $(30 \%, \mathrm{aOR}=3.1,95 \% \mathrm{CI}: 1.1-8.4)$ were more likely to report vaccine hesitancy compared to mothers with a graduate degree (19\% hesitant; Table 1). NonHispanic Black mothers (40\% hesitant, aOR $=2.8,95 \% \mathrm{CI}$ : 1.0-7.6) were more likely to be vaccine hesitant compared to non-Hispanic white mothers (19\% hesitant); mothers identifying as non-Hispanic Asian, Hispanic and other races/ ethnicities did not differ in vaccine hesitancy compared to non-Hispanic white mothers when controlling for education and pandemic-related anxiety (Table 1). Mothers with low pandemic-related anxiety were more likely to report vaccine hesitancy compared to those with high pandemicrelated anxiety ( $56 \%$ vs $23 \%$ hesitant; aOR $=4.8,95 \% \mathrm{CI}$ : 1.7-14.1); low-to-medium and medium-to-high anxiety were not associated with vaccine hesitancy (Table 1).

\section{Discussion}

When surveyed in mid-February to mid-March 2021, 28\% of unvaccinated mothers of children 0-12 years old in our sample reported that they would probably not or definitely not get a COVID-19 vaccine, similar to rates of vaccine hesitancy in US adults of $30 \%$ during that time frame (Funk \& Tyson, 2021). We found that mothers with a high school, trade, or technical education, those who had earned an Associate's degree, and those with a Bachelor's degree were more likely to be vaccine hesitant than mothers with a graduate degree, similar to differences by education observed among US adults generally (Khubchandani et al., 2021; Nguyen et al., 2021).

We found that non-Hispanic Black mothers were more likely to be vaccine hesitant than non-Hispanic white mothers (40\% vs 19\%), a difference not explained by education or pandemic-related anxiety. This finding is consistent with previous research documenting higher rates of COVID-19 vaccine hesitancy in Black adults compared to white adults, with rates of hesitancy related to medical distrust, experiences of racial discrimination, and exposure to misinformation related to COVID-19 or the vaccines (Khubchandani \& Macias, 2021). Hispanic/Latina mothers in our sample were more likely to be vaccine hesitant than non-Hispanic white mothers (39\% vs $19 \%)$, but this difference was no longer statistically significant after controlling for other factors. While Native communities have also been disproportionally impacted by the COVID-19 pandemic (Mackey et al., 2021), only 12 women in our sample identified as Native, limiting our ability to examine their rates of vaccine hesitancy.

We also found that mothers with low pandemic-related anxiety were more likely to be vaccine hesitant than those with high pandemic-related anxiety ( $56 \%$ vs $23 \%$ ), consistent with national surveys of US adults in May 2020 (Head et al., 2020) and December 2020 (Nguyen et al., 2021) that also found greater concern or worry about COVID-19 infection was associated with vaccine intentions. A global survey of pregnant women and non-pregnant mothers in late fall 2020 also found that women worried about COVID-19 were more likely to intend to get vaccinated than women who were not worried (Skjefte et al., 2021).

While mothers who had a close family member test positive for COVID-19 were more likely to be vaccine hesitant (40\% vs 22\%), this difference was accounted for by differences in other factors associated with vaccine hesitancy. The majority of COVID-19 infections are asymptomatic or mild, especially among younger and middle-aged adults (Wiersinga, et al., 2020), and it may be that having a family member experience a mild disease course results in mothers weighing the unknown risks of vaccination against their experiences with COVID-19. However, neither personal infection nor knowing someone personally who died of COVID-19 was associated with vaccine hesitancy in our sample. As older adults are more likely to experience severe disease and have higher death rates (Wiersinga et al., 2020), we expected that mothers living with someone aged 65 years or older would be less likely to be vaccine hesitant. While only $9 \%$ of our sample $(n=19)$ had an elderly housemate, limiting our power to detect differences, the hesitancy rates (11\% vs $29 \%$ ) observed in this study suggest exploring in larger samples whether vaccine intentions differs by presence of an elderly individual or other person at elevated risk in one's household. While not statistically significant, our finding that $36 \%$ of women from small cities or towns or rural areas and $24 \%$ of women from large cities or suburbs 
near large cities would probably not or definitely not get a COVID-19 vaccine is similar to rates of vaccine hesitancy in previous research that found differences by urbanicity/rurality of residence among US adults generally (Khubchandani et al., 2021; Nguyen et al., 2021). Despite a lack of clinical trial data about vaccine safety and efficacy in pregnant persons and misinformation circulating online about fertility-related vaccine side effects, leading to uncertainty and concerns about vaccination during pregnancy (Brillo, et al., 2021), vaccine hesitancy did not differ by pregnancy status in the current study (33\% vs $27 \%$ ), consistent with a global study of pregnant women and non-pregnant mothers in fall 2020 (Skjefte et al., 2021). In early 2021, an observational study showed similar immunogenicity and reactogenicity to a mRNA COVID-19 vaccine in pregnant and non-pregnant women (Gray et al., 2021), and the American College of Obstetricians and Gynecologists and the Society for Maternal-Fetal Medicine recommend vaccination for pregnant women (ACOG, 2020; SMFM, 2020).

This study has additional strengths and limitations. Our sample was racially/ethnically and geographically diverse, but more highly educated than US mothers overall (Matthews \& Hamilton, 2019). As all participants had at least a high school education, and few did not have at least college coursework, the current study does not provide insights into COVID-19 vaccine hesitancy among women with low educational attainment. Our sample were all active social media users, compared to about $80 \%$ of women aged $18-49$ years in the US, so our sample may underrepresent mothers less likely to use social media (e.g., those from rural areas) (Auxier \& Anderson, 2021). We had limited statistical power to detect differences between certain groups (e.g., pregnant women, residents of rural areas, those with an elderly household member).

COVID-19 vaccine hesitancy is multi-faceted, and includes worry about infection, concerns about vaccine safety or efficacy, distrust of scientific research, emotional responses such as fear, and general anti-vaccination attitudes (Chou \& Budenz, 2020; Funk \& Tyson, 2021; Head et al., 2020; Khubchandani \& Macias, 2021; Skjefte et al., 2021). Research is needed to understand informational, emotional, and attitudinal factors contributing to COVID-19 vaccine hesitancy among mothers and how these factors interact with structural barriers impacting vaccine access (Hunter et al., 2021). Research is also needed to develop and test effective public health messaging to increase vaccination rates among mothers, building on existing recommendations for increasing vaccine confidence and vaccination rates which include using jargon-free language, acknowledging and addressing people's concerns, tailoring messages for specific audiences, preventing the spread of misinformation, and engaging community organizations and trusted community leaders (Hunter et al., 2021; Khubchandani \& Macias,
2021; National Academies of Sciences \& Medicine, 2021). In addition to increasing COVID-19 vaccination rates among US adults, overcoming maternal vaccine hesitancy may also contribute to achieving high rates of vaccination among children, as mothers who have received or intend to receive a COVID-19 vaccine are substantially more likely to intend to vaccinate their children (Szilagyi et al., 2021), highlighting the importance of understanding and addressing maternal vaccine hesitancy.

Author contributions Conception of the work (Waring, Pagoto), acquisition of data (Waring, Rudin, Ho, Horkachuck, Kapoor, Foye), analysis of data (Waring), interpretation of data (all authors), drafted the work (Waring, Pagoto, Rudin), revised work critically for important intellectual content (all authors), approved the version to be published (all authors).

Funding This work was supported by USDA National Institute of Food and Agriculture, Hatch project 1020701. Additional support for SLP provided by NIH grant K24HL124366.

\section{Declarations}

Conflict of interest Dr. Pagoto provides consulting for Neutrogena which is a brand of the Johnson \& Johnson Family of Consumer Companies. The other authors declare that they have no conflicts of interest.

Ethics approval All procedures performed in studies involving human participants were in accordance with the ethical standards of the institutional and/or national research committee and with the 1964 Helsinki declaration and its later amendments or comparable ethical standards. The University of Connecticut Institutional Review Board approved this study.

Human and Animal Rights and Informed Consent All procedures followed were in accordance with ethical standards of the responsible committee on human experimentation (institutional and national) and with the Helsinki Declaration of 1975, as revised in 2000. Informed consent was obtained from all participants.

Consent to participate All participants provided informed consent.

\section{References}

American College of Obstetricians \& Gynecologists (ACOG). (2020). COVID-19 vaccination considerations for obstetric-gynecologic care. Retrieved from https://www.acog.org/clinical/clinical-guida nce/practice-advisory/articles/2020/12/covid-19-vaccinationconsiderations-for-obstetric-gynecologic-care

Auxier, B., \& Anderson, M. (2021). Social Media use in 2021. Retrieved from https://www.pewresearch.org/internet/2021/04/ 07/social-media-use-in-2021/

Brillo, E., Tosto, V., Gerli, S., \& Buonomo, E. (2021). COVID-19 vaccination in pregnancy and postpartum. The Journal of MaternalFetal \& Neonatal Medicine. https://doi.org/10.1080/14767058. 2021.1920916 
Chou, W. S., \& Budenz, A. (2020). Considering emotion in COVID-19 vaccine communication: Addressing vaccine hesitancy and fostering vaccine confidence. Health Communication, 35, 1718-1722. https://doi.org/10.1080/10410236.2020.1838096

Funk, C., \& Tyson, A. (2021). Growing share of Americans say they plan to get a COVID-19 vaccine-or already have. Retrieved from https://www.pewresearch.org/science/2021/03/05/growing-shareof-americans-say-they-plan-to-get-a-covid-19-vaccine-or-alrea dy-have/

Gomez, I., Ranji, U., \& Salganicoff, A. (2018). Women, work, and family health: Key findings from the 2017 Kaiser women's health survey. Retrieved from https://www.kff.org/womens-healthpolicy/issue-brief/women-work-and-family-health-key-findi ngs-from-the-2017-kaiser-womens-health-survey/

Gray, K. J., Bordt, E. A., Atyeo, C., Deriso, E., Akinwunmi, B., Young, N., Baez, A. M., Shook, L. L., Cvrk, D., James, K., De Guzman, R., Brigida, S., Diouf, K., Goldfarb, I., Bebell, L. M., Yonker, L. M., Fasano, A., Rabi, S. A., Elovitz, M. A., Alter, G. \& Edlow, A. G. (2021). Coronavirus disease 2019 vaccine response in pregnant and lactating women: A cohort study. American Journal of Obstetrics and Gynecology, 225, 303.e301-303.e317. https://doi. org/10.1016/j.ajog.2021.03.023

Harris, P. A., Scott, K. W., Lebo, L., Hassan, N., Lightner, C., \& Pulley, J. (2012). ResearchMatch: A national registry to recruit volunteers for clinical research. Academic Medicine, 87, 66-73. https://doi. org/10.1097/ACM.0b013e31823ab7d2

Head, K. J., Kasting, M. L., Sturm, L. A., Hartsock, J. A., \& Zimet, G. D. (2020). A national survey assessing SARS-CoV-2 vaccination intentions: Implications for future public health communication efforts. Science Communication, 42, 698-723. https://doi.org/10. 1177/1075547020960463

Hunter, C. M., Chou, W. S., \& Webb Hooper, M. (2021). Behavioral and social science in support of SARS-CoV-2 vaccination: National Institutes of Health initiatives. Transl Behav Med. https:// doi.org/10.1093/tbm/ibab067

Khubchandani, J., \& Macias, Y. (2021). COVID-19 vaccination hesitancy in Hispanics and African-Americans: A review and recommendations for practice. Brain, Behavior, and Immunity Health, 15, 100277. https://doi.org/10.1016/j.bbih.2021.100277

Khubchandani, J., Sharma, S., Price, J. H., Wiblishauser, M. J., Sharma, M., \& Webb, F. J. (2021). COVID-19 Vaccination hesitancy in the United States: A rapid national assessment. Journal of Community Health, 46, 270-277. https://doi.org/10.1007/s10900-020-00958-x

Mackey, K., Ayers, C. K., Kondo, K. K., Saha, S., Advani, S. M., Young, S., Spencer, H., Rusek, M., Anderson, J., Veazie, S., Smith, M., \& Kansagara, D. (2021). Racial and ethnic disparities in COVID-19-related infections, hospitalizations, and deaths: A systematic review. Annals of Internal Medicine, 174, 362-373. https://doi.org/10.7326/m20-6306

Matthews, T. J., \& Hamilton, B. E. (2019). Educational attainment of mothers aged 25 and over: United States, 2017. NCHS Data Brief, 332, 1-8.

McElroy, E., Patalay, P., Moltrecht, B., Shevlin, M., Shum, A., Creswell, C., \& Waite, P. (2020). Demographic and health factors associated with pandemic anxiety in the context of COVID-19.
British Journal of Health Psychology, 25, 934-944. https://doi. org/10.1111/bjhp. 12470

Murthy, B. P., Sterrett, N., Weller, D., Zell, E., Reynolds, L., Toblin, R. L., Murthy, N., Kriss, J., Rose, C., Cadwell, B., Wang, A., Ritchey, M. D., Gibbs-Scharf, L., Qualters, J. R., Shaw, L., Brookmeyer, K. A., Clayton, H., Eke, P., Adams, L., Zajac, J., Patel, A., Fox, K., Williams, C., Stokley, S., Flores, S., Barbour, K. E. \& Harris, L. Q. (2021). Disparities in COVID-19 vaccination coverage between urban and rural counties-United States, December 14, 2020-April 10, 2021. MMWR Morbidity and Mortality Weekly Report, 70, 759-764. https://doi.org/10.15585/mmwr.mm7020e3

National Academies of Sciences, E., \& Medicine. (2021). Strategies for building confidence in the COVID-19 Vaccines. The National Academies Press. https://www.nap.edu/catalog/26068/strategiesfor-building-confidence-in-the-covid-19-vaccines

Nguyen, K. H., Srivastav, A., Razzaghi, H., Williams, W., Lindley, M. C., Jorgensen, C., Abad, N., \& Singleton, J. A. (2021). COVID-19 vaccination intent, perceptions, and reasons for not vaccinating among groups Prioritized for early vaccination-United States, September and December 2020. MMWR Morbidity and Mortality Weekly Report, 70, 217-222. https://doi.org/10.15585/mmwr. mm7006e3

Powers, B. J., Trinh, J. V., \& Bosworth, H. B. (2010). Can this patient read and understand written health information? JAMA, 304, 76-84. https://doi.org/10.1001/jama.2010.896

Skjefte, M., Ngirbabul, M., Akeju, O., Escudero, D., HernandezDiaz, S., Wyszynski, D. F., \& Wu, J. W. (2021). COVID-19 vaccine acceptance among pregnant women and mothers of young children: Results of a survey in 16 countries. European Journal of Epidemiology, 36, 197-211. https://doi.org/10.1007/ s10654-021-00728-6

Society for Maternal-Fetal Medicine (SMFM). (2020). Society for Maternal-Fetal Medicine (SMFM) statement: SARS-CoV-2 vaccination in pregnancy. Retrieved from https://s3.amazonaws.com/ cdn.smfm.org/media/2591/SMFM_Vaccine_Statement_12-1-20_ (final).pdf

Szilagyi, P. G., Shah, M. D., Delgado, J. R., Thomas, K., Vizueta, N., Cui, Y., Vangala, S., Shetgiri, R., \& Kapteyn, A. (2021). Parents' intentions and perceptions about COVID-19 vaccination for their children: Results from a national survey. Pediatrics, 148. https://doi.org/10.1542/peds.2021-052335

US Census Bureau. (2021). Household pulse survey: Measuring social and economic impacts during the coronavirus pandemic. Retrieved from https://www2.census.gov/programs-surveys/ demo/technical-documentation/hhp/Phase\%203\%20Question naire_02.25.21_English.pdf

Wiersinga, W. J., Rhodes, A., Cheng, A. C., Peacock, S. J., \& Prescott, H. C. (2020). Pathophysiology, transmission, diagnosis, and treatment of coronavirus disease 2019 (COVID-19): A review. JAMA, 324, 782-793. https://doi.org/10.1001/jama.2020.12839

Publisher's Note Springer Nature remains neutral with regard to jurisdictional claims in published maps and institutional affiliations. 